\title{
A note on involution centralizers in black box groups
}

\author{
Veronica Kelsey and Peter Rowley \\ Communicated by Michael Giudici
}

\begin{abstract}
Here we note a minor variation on the method in [J. N. Bray, An improved method for generating the centralizer of an involution, Arch. Math. (Basel) 74 (2000), no. 4, 241-245] which enables calculations of $C_{H}(t)$ for $H$ a subgroup of a black box group $G$ and $t$ an involution of $G$. We then give some applications of this, the first to investigate the action of the Lyons sporadic group via conjugation on its involutions, a number of calculations in $E_{6}(2)$ and $E_{8}(2)$, then finally to carry out some calculations in $\mathrm{GL}_{670}(2)$.
\end{abstract}

\section{Introduction}

In [7], Bray revealed a method for calculating centralizers of involutions in black box groups with an order oracle. This method extended one introduced earlier by R. Parker (see [18]). In recent times, the Bray method has had many ramifications in computational group theory (for a fraction of these, consult $[2,6,8,11-14,19$, $20,25,26])$. The purpose of this short note is to observe a further twist to this story. Although this perturbation to [7] is very minor, it results in some substantial gains. In Section 2, we make use of it to determine the suborbits of the Lyons sporadic simple group in its action by conjugation on its involutions. A further demonstration of the efficacy of this variation, in Section 2.4, are some calculations in $\mathrm{GL}_{670}(2)$. Also in Section 2, we include statistics of some sample calculations in $E_{6}(2)$ and $E_{8}(2)$.

Suppose $G$ is a black box group with an order oracle. Assume $t$ is an involution of $G$. In [7], the elements $\mathcal{K}(t, g)$ of $G$ are key. For $g \in G$ and letting $n$ be the order of $[t, g]$, we define

$$
\mathcal{K}(t, g)= \begin{cases}{[t, g]^{m}} & \text { if } n=2 m, \\ g[t, g]^{m} & \text { if } n=2 m+1 .\end{cases}
$$

These elements $\mathcal{K}(t, g)$ (and also $\mathcal{K}\left(t, g^{-1}\right)$ if $n$ is even) supply elements in $C_{G}(t)$. Those $\mathcal{K}(t, g)$ obtained when $n$ is odd have the property observed by R. Parker that they are uniformly distributed throughout $C_{G}(t)$. 
For $H \leq G$, set $\mathcal{O}_{H}=\{h \mid h \in H$ and $[t, h]$ is of odd order $\}$.

Lemma 1.1. Suppose $t$ is an involution in $G, H \leq G$, and let $c \in C_{H}(t)$. Then $\left|\left\{h \in \mathcal{O}_{H} \mid \mathcal{K}(t, h)=c\right\}\right|$ is independent of $c$.

Proof. Since $c \in C_{H}(t),[t, h]=[t, c h]$ for all $h \in H$, so we only need prove the lemma for each right $\operatorname{coset} C_{H}(t) h(h \in H)$ for which $[t, h]$ has odd order. For $e h \in C_{H}(t) h$, we have

$$
\mathcal{K}(t, e h)=e h[t, e h]^{m}=e h[t, h]^{m}=e \mathcal{K}(t, h),
$$

where $[t, h]$ has order $2 m+1$. Hence each such coset contributes 1 to

$$
\left|\left\{h \in \mathcal{O}_{H} \mid \mathcal{K}(t, h)=c\right\}\right|,
$$

so giving the lemma.

Theorem 3.1 of [7] is the case $H=G$, and its proof is virtually identical to that for Lemma 1.1. The point is that $t$ does not need to be in $H$. So to compute $C_{H}(t)$, we may proceed as follows.

(1) Initialize $S:=\{\}$.

(2) Choose a random element $h \in H$.

(3) Compute $\mathcal{K}(t, h)$.

(4) Check whether $\mathcal{K}(t, h) \in H$; if yes, then add $\mathcal{K}(t, h)$ to $S$.

(5) Go to step (2).

Then $\langle S\rangle$ will be a subgroup of $C_{H}(t)$. All the analysis and caveats discussed in [7] will apply here. Lemma 1.1 shows that the set of elements passing test (4) will be uniformly distributed in $C_{H}(t)$. Also, the membership problem raises its head in step (4), and the exact nature of $H$ may help in resolving this. For example, we may have $H=C_{G}(X)(X \subseteq G)$ in which case step (4) can be settled by checking whether $\mathcal{K}(t, h)$ commutes with the set $X$. The other conundrum facing us is what is the probability that the elements $\mathcal{K}(t, h)$ are actually in $H$. Without further specific information about $H$, there does not seem much that can be said in general. Indeed, we could have that $C_{H}(t)=1$. In this connection, in Section 2, we gather statistics of some actual calculations.

Suppose $H=C_{G}(s)$, where $s$ is an involution of $G$ (in fact, the situation that sparked this note); experimentally, the following works well. In place of (2)-(5), do the following. 
(2') Compute $\mathcal{K}(s, g)$, where $g$ is a random element of $G$ (so applying the Bray method for $\left.C_{G}(s)\right)$.

$\left(3^{\prime}\right)$ Compute $\mathcal{K}(t, \mathcal{K}(s, g))$.

(4') Check whether $s$ and $\mathcal{K}(t, \mathcal{K}(s, g))$ commute; if yes, then add $\mathcal{K}(t, \mathcal{K}(s, g))$ to $S$.

$\left(5^{\prime}\right)$ Go to step $\left(2^{\prime}\right)$.

Observe that Lemma 1.1 applied twice shows that these will be uniformly distributed in $C_{G}(t) \cap C_{G}(s)$. Of course, we could determine generating sets for $C_{G}(t)$ and $C_{G}(s)$ using the Bray method, and then we could attempt to compute $C_{G}(t) \cap C_{G}(s)$. In computationally hard groups, the latter step may prove impossible. To illustrate this with an example, take $G=E_{6}(2)$, as given in the electronic ATLAS [24] in its 27-dimension GF(2) representation. There $G=\langle a, b\rangle$, where $a$ has order $2, b$ has order 3 with $a b$ of order 62 . Taking $t=a$ and $s=(a b)^{31}$, using the method discussed here (that is, steps $\left.(1),\left(2^{\prime}\right),\left(3^{\prime}\right),\left(4^{\prime}\right),\left(5^{\prime}\right)\right)$ with 10,000 random elements $g \in G$, we get $\langle S\rangle=C_{G}(s) \cap C_{G}(t)$ with $|\langle S\rangle|=2^{12} \cdot 3 \cdot 7$. This is done in the blink of an eye, whereas first calculating $C_{G}(s)$ and $C_{G}(t)$ (which is quick) and then $C_{G}(s) \cap C_{G}(t)$ takes forever $(1552$ seconds on a $16 \times 1248 \mathrm{MHz}$ machine running MAGMA [9] version 222-10). This disparity will be even greater for larger groups.

Finally, we observe that the above process for the centralizer of two involutions may be iterated so as to find $C_{G}(H)$, where $H$ is generated by involutions - see Section 2.4 for an example of this.

\section{Some applications}

\subsection{Involutions in the Lyons group}

The sporadic simple group Ly, first pin-pointed by Lyons in [15], has order $2^{8} \cdot 3^{7}$. $5^{6} \cdot 7 \cdot 11 \cdot 31 \cdot 37 \cdot 67$. Its smallest non-trivial permutation degree is $8,835,156-$ indeed, this representation was built in the 1970s by Sims [22] in the course of showing the existence of Ly. Despite considerable advances in computer algebra, such a permutation representation is not viable for probing the structure of Ly. More tractable is the 111-dimensional representation over GF(5), first displayed in [17], which we employ here. From [24], we obtain this representation where Ly is generated by the standard generators $a, b$ with $a \in 2 A, b \in 5 A$ and $a b \in 14 A$ (using ATLAS [10] names for the conjugacy classes of Ly).

For many of the sporadic simple groups $G$, the action (by conjugation) of $G$ on a $G$-conjugacy class of involutions has been analyzed. See $[4,5,21,23]$, where the 
suborbits are determined. This type of information is valuable when studying such topics as commuting involution graphs [3], $C$-strings and polytopes [16].

Here we investigate the action of $G \cong$ Ly upon $X=2 A$. Before stating our main conclusion, we introduce two pieces of notation. Let $t \in X$. For $C$ a $G$-conjugacy class, $X_{C}=\{x \in X \mid t x \in C\}$, and for $y \in G$,

$$
C_{G}^{*}(y)=\left\{g \in G \mid y^{g}=y \text { or } y^{g}=y^{-1}\right\} .
$$

Observe that $X_{C}$ will either be empty or the union of $C_{G}(t)$-orbits (under conjugation) and, of course, $X=\dot{\cup} X_{C}$, as $C$ runs through all the $G$-conjugacy classes. As is well known, $\left|X_{C}\right|$ equals a particular structure constant which may be calculated using the complex irreducible character table of $G$. Also, we have $\left[C_{G}^{*}(y): C_{G}(y)\right]=1$ or 2 .

The following easy lemma will be used in the proof of Theorem 2.2.

Lemma 2.1. For $t, x \in X$, put $z=t x$. Then

$$
C_{C_{G}(t)}(x)=C_{G}(t) \cap C_{G}(x)=C_{C_{G}(z)}(t)=C_{C_{G}(z)}(x) .
$$

So, for $z=t x \in C$, where $x \in X$ if we know the structure of $C_{G}(z)$, we may be able to calculate $C_{G}(t) \cap C_{G}(x)$. In particular, note that $\left[C_{G}^{*}(z): C_{G}(z)\right]=2$ and, as $G=$ Ly has just one conjugacy class of involutions, $C_{C_{G}(z)}(y)$ as $y$ ranges through all involutions in $C_{G}^{*}(z) \backslash C_{G}(z)$ gives all the possible structures for

$$
C_{G}(t) \cap C_{G}(y), \quad y \in X_{C},
$$

that must occur (but not the possible multiplicities).

As we shall see, a number of the $X_{C}$ may be easily broken into $C_{G}(t)$-orbits. However, for three $G$-conjugacy classes, it is not so straightforward, and we make use of the routine $(1),\left(2^{\prime}\right),\left(3^{\prime}\right),\left(4^{\prime}\right),\left(5^{\prime}\right)$ given in Section 1.

Theorem 2.2. Suppose that $G=\mathrm{Ly}, X=2 A$ and $t \in X$. Then the permutation rank of $G$ on $X$ is 78 and the suborbit (that is, $C_{G}(t)$-orbit) sizes are as follows.

\begin{tabular}{lrr}
\hline$C$ & $\left|X_{C}\right|$ & $C_{G}(t)$-orbit sizes \\
\hline $1 A$ & 1 & 1 \\
$2 A$ & 34,650 & 34,650 \\
$3 A$ & 5,040 & 5,040 \\
$3 B$ & 677,600 & $554,400+123,200$ \\
\hline
\end{tabular}




\begin{tabular}{|c|c|c|}
\hline$C$ & $\left|X_{C}\right|$ & $C_{G}(t)$-orbit sizes \\
\hline $4 A$ & $1,663,200$ & $831,600+831,600$ \\
\hline $5 A$ & 199,584 & 299,584 \\
\hline $5 B$ & $5,322,240$ & $1,330,560+3,991,680$ \\
\hline $6 A$ & 831,600 & 831,600 \\
\hline $6 B$ & $6,098,400$ & $1,108,800+4,989,600$ \\
\hline $6 C$ & $13,305,600$ & $3,326,400+3,326,400+3,326,400+3,326,400$ \\
\hline $7 A$ & $19,958,400$ & $19,958,400$ \\
\hline $8 A$ & 979,200 & $4,989,600+4,989,600$ \\
\hline $8 B$ & $19,958,400$ & $9,979,200+9,979,200$ \\
\hline $9 A$ & $26,611,200$ & $19,958,400+6,652,800$ \\
\hline $10 A$ & $4,989,600$ & $4,989,600$ \\
\hline $10 B$ & $39,916,800$ & $19,958,400+19,958,400$ \\
\hline $12 A$ & $14,968,800$ & $4,989,600+9,979,200$ \\
\hline $12 B$ & $26,611,200$ & $6,652,800+19,958,400$ \\
\hline $14 A$ & $19,958,400$ & $19,958,400$ \\
\hline $15 A$ & $7,983,360$ & $1,995,840+1,995,840+1,995,840+1,995,840$ \\
\hline $15 B$ & $39,916,800$ & $19,958,400+19,958,400$ \\
\hline $15 C$ & $39,916,800$ & $39,916,800$ \\
\hline $18 A$ & $39,916,800$ & $19,958,400+19,958,400$ \\
\hline $20 A$ & $39,916,800$ & $19,958,400+19,958,400$ \\
\hline $21 A$ & $39,916,800$ & $19,958,400+19,958,400$ \\
\hline $21 B * *$ & $39,916,800$ & $19,958,400+19,958,400$ \\
\hline $24 A$ & $39,916,800$ & $19,958,400+19,958,400$ \\
\hline $24 B$ & $39,916,800$ & $19,958,400+19,958,400$ \\
\hline $24 C *$ & $39,916,800$ & $19,958,400+19,958,400$ \\
\hline $25 A$ & $39,916,800$ & $39,916,800$ \\
\hline $28 A$ & $39,916,800$ & $19,958,400+19,958,400$ \\
\hline $30 A$ & $39,916,800$ & $19,958,400+19,958,400$ \\
\hline $30 B$ & $39,916,800$ & $19,958,400+19,958,400$ \\
\hline $31 A$ & $39,916,800$ & $39,916,800$ \\
\hline $31 B * 2$ & $39,916,800$ & $39,916,800$ \\
\hline
\end{tabular}




\begin{tabular}{lrr}
\hline$C$ & $\left|X_{C}\right|$ & $C_{G}(t)$-orbit sizes \\
\hline $31 C * 4$ & $39,916,800$ & $39,916,800$ \\
$31 D * 8$ & $39,916,800$ & $39,916,800$ \\
$31 E * 16$ & $39,916,800$ & $39,916,800$ \\
$37 A$ & $39,916,800$ & $39,916,800$ \\
$37 B *$ & $39,916,800$ & $39,916,800$ \\
$40 A$ & $39,916,800$ & $19,958,400+19,958,400$ \\
$40 B *$ & $39,916,800$ & $19,958,400+19,958,400$ \\
$42 A$ & $39,916,800$ & $19,958,400+19,958,400$ \\
$42 B *$ & $39,916,800$ & $19,958,400+19,958,400$ \\
$67 A$ & $39,916,800$ & $39,916,800$ \\
$67 B * 2$ & $39,916,800$ & $39,916,800$ \\
$67 C * 4$ & $39,916,800$ & $39,916,800$ \\
\hline
\end{tabular}

Proof. Let $x \in X$, and set $z=t x \in C, C$ a $G$-conjugacy class. We recall that $C_{G}(t) \cong 2 \cdot \operatorname{Alt}(11)$, so $\left|C_{G}(t)\right|=39,916,800=2^{8} \cdot 3^{4} \cdot 5^{2} \cdot 7 \cdot 11$.

Suppose that $C_{G}(z)=\langle z\rangle$. Then if $z$ has odd order, $C_{G}(t) \cap C_{G}(y)=1$ for all $y \in X_{C}$, and if $z$ has even order, $\left|C_{G}(t) \cap C_{G}(y)\right|=2$ for all $y \in X_{C}$. This deals with $X_{C}$ for

$$
C \in\left\{\begin{array}{l}
18 A, 24 A, 24 B, 24 C *, 25 C, 28 A, 31 A, 31 B * 2,31 C * 4,31 D * 8, \\
31 E * 16,37 A, 37 B *, 40 A, 40 B *, 42 A, 42 B *, 67 A, 67 B * 2,67 C * 4
\end{array}\right\} .
$$

Also if $z$ has odd order and $\left[C_{G}(z):\langle z\rangle\right]=2$, then $\left|C_{G}(t) \cap C_{G}(y)\right|=2$ for all $y \in X_{C}$. This observation yields that $X_{21 A}$ and $X_{21 B *}$ both break into two $C_{G}(t)$ orbits.

If $z$ has even order, then, as $G$ has only one involution conjugacy class, we may regard $C_{G}(t) \cap C_{G}(x)$ as a subgroup of $2 \cdot \operatorname{Alt}(11)$. So we may calculate in 2 Alt(11), using the permutation representation of degree 5,040 in [24]. This approach allows us to break $X_{C}$ into $C_{G}(t)$-orbits for

$$
C \in\{2 A, 4 A, 6 A, 6 B, 6 C, 8 A, 8 B, 10 A, 10 B, 12 A, 12 B, 14 A, 20 A\} .
$$

Note that, for $z \in 7 A, C_{G}^{*}(z)=C_{G}^{*}(w)$, where $w^{2}=z$ and $w \in 14 A$, so $X_{7 A}$ is a $C_{G}(t)$-orbit just as $X_{14 A}$ is.

At this point, it remains to examine $X_{C}$ for

$$
C \in\{3 A, 3 B, 5 A, 5 B, 9 A, 15 A, 15 B, 15 C\} .
$$


For $z \in C=3 A, C_{G}^{*}(z) \sim 3 \cdot \mathrm{McL}: 2$ and $C_{C_{G}(z)}(t) \cong M_{11}$ (see [10]), and therefore $X_{3 A}$ is a $C_{G}(t)$-orbit. Suppose $z$ has order 15. For $z \in 15 B$, we have $C_{G}^{*}(z)=C_{G}(w)$ for $w \in 30 B$ and $w^{2}=z$, so $X_{15 B}$ breaks into two $C_{G}(t)$ orbits just as $X_{30 B}$ does. If $z \in 15 A \cup 15 C$, then $z^{5} \in 3 A$, and we may calculate in McL : 2, using the permutation representation of McL : 2 of degree 275. Suppose $z \in 9 A$. Then $\left[C_{G}(z):\langle z\rangle\right]=6$. Since $G$ has no elements of order 18 , $C_{G}(z) /\langle z\rangle$ cannot be cyclic, and so $C_{G}(z) /\langle z\rangle \cong \operatorname{Sym}(3)$. If $y$ is an involution inverting $z$, then $y$ must induce inner automorphisms on $C_{G}(z) /\langle z\rangle$. Since $C_{C_{G}(z)}(y) \cap\langle z\rangle=1$, we conclude that $C_{C_{G}(z)}(y)=2$ or 6 ; whence it follows that $X_{9 A}$ had two $C_{G}(t)$-orbits of sizes $19,958,400$ and $6,652,800$.

We are now left with the cases $C \in\{3 B, 5 A, 5 B\}$. The structure of $C_{G}^{*}(z)$ in these cases is more intricate, so we use the procedure discussed in Section 1.

(2.2.1) $C=3 B$. For different choices of $x_{1}, x_{2} \in X_{3 B}$, we obtain

$$
L_{i} \leq C_{G}(t) \cap C_{G}\left(x_{i}\right),
$$

where $\left|L_{1}\right|=2^{2} \cdot 3^{4},\left|L_{2}\right|=2^{3} \cdot 3^{2}$. In fact, $L_{i}=C_{G}(t) \cap C_{G}\left(x_{i}\right)$ for $i=1,2$, and we explain how this was established for $i=1, i=2$ being similar. Using the LMGRadicalQuotient command in MAGMA on $C_{G}(t) \cong 2 \cdot \operatorname{Alt}(11)$, we obtain a map from $C_{G}(t)$ to $C_{G}(t) /\langle t\rangle \cong \operatorname{Alt}(11)$, with the latter given as a permutation group of degree 11. Let $\overline{L_{1}}$ be the projection of $L_{1}$ into Alt(11) (and note $L_{1} \cap\langle t\rangle=1$ ). Because $\left|\overline{L_{1}}\right|=3^{4} \cdot 2^{2}$, the only possible maximal subgroups of Alt(11) containing $\overline{L_{1}}$ are isomorphic to either $\operatorname{Sym}(9)$ or Alt(10). Within Alt(10), the only proper overgroups might be Alt(9), and then within Alt(9) only $3^{3}: \operatorname{Sym}(4)$. Within $\operatorname{Sym}(9)$, the only proper subgroup that could contain $\overline{L_{1}}$ has shape $3^{3}:(\operatorname{Sym}(4) \times 2)$. So if $C_{G}(t) \cap C_{G}\left(x_{1}\right)>L_{1}$, we must have $2^{3}$ dividing $\left|C_{G}(t) \cap C_{G}\left(x_{1}\right)\right|$. Selecting a Sylow 2 -subgroup of $\overline{L_{1}}, \overline{S_{1}}$, we calculate $N_{C_{G}(t) /\langle t\rangle}\left(\overline{S_{1}}\right)$ and then take its full inverse image in $C_{G}(t)$, say $K$. Since we have $|K|=2^{6} \cdot 3$, we may quickly enumerate $C_{K}\left(x_{1}\right)$ finding that $\left|C_{K}\left(x_{1}\right)\right|=2^{2}$. So $2^{3}$ does not divide $\left|C_{G}(t) \cap C_{G}\left(x_{1}\right)\right|$, and we conclude that

$$
C_{G}(t) \cap C_{G}\left(x_{1}\right)=L_{1} .
$$

This gives a $2^{6} \cdot 5^{2} \cdot 7 \cdot 11=123,200$ size $C_{G}(t)$-orbit of $X_{3 B}$.

(2.2.2) $\boldsymbol{C}=\mathbf{5} \boldsymbol{A}$. Selecting a random $x \in X_{5 A}$, we use the procedure in Section 1 and obtain $L \leq C_{G}(t) \cap C_{G}(x)$ with $L \sim 5^{2}: 2$. When taking the image of $L$ (as for $X_{3 B}$ ), say $\bar{L}$, in $C_{G}(t) /\langle t\rangle \cong \operatorname{Alt}(11)$, we find

$$
\bar{L} \leq \bar{K} \cong(\operatorname{Alt}(5) \times \operatorname{Alt}(5)) \cdot 4 .
$$


Taking the full inverse image of $\bar{K}$, say $K$, in $C_{G}(t)$ (which has order $2^{8} \cdot 3^{2} \cdot 5^{2}$ ), we calculate that $\left|K \cap C_{G}(x)\right|=2^{3} \cdot 5^{2}$. Projecting $K \cap C_{G}(x)$ back into Alt(11), we see that $K \cap C_{G}(x)=C_{G}(t) \cap C_{G}(x)$. Consequently, $X_{5 A}$ consists of a single $C_{G}(t)$-orbit.

(2.2.3) $\boldsymbol{C}=\mathbf{5 B}$. Again selecting a random $x \in X_{5 B}$ and employing the procedure in Section 1 , we locate $x_{1}, x_{2} \in X_{5 B}$ with

$$
L_{1} \leq C_{G}(t) \cap C_{G}\left(x_{1}\right), \quad L_{2} \leq C_{G}(t) \cap C_{G}\left(x_{2}\right),
$$

$L_{1} \cong \operatorname{Dih}(10)$ and $L_{2} \cong \operatorname{Dih}(30)$. Let $\overline{L_{1}}$ be the image of $L_{1}$ in $C_{G}(t) /\langle t\rangle$, and let $\overline{N_{2}}$ and $\overline{N_{5}}$ be the normalizers in Alt(11) of respectively a Sylow 2- and 5-subgroup of $\overline{L_{1}}$. If $N_{2}$ and $N_{5}$ are respective full inverse images of $\overline{N_{2}}$ and $\overline{N_{5}}$, we have $\left|N_{2}\right|=2 \cdot 1,152$ and $\left|N_{5}\right|=2 \cdot 100$; whence we quickly check that

$$
\left|N_{2} \cap C_{G}(x)\right|=2 \text { and }\left|N_{5} \cap C_{G}(x)\right|=10 .
$$

Hence we conclude that $C_{G}(t) \cap C_{G}\left(x_{1}\right)=L_{1} \cong \operatorname{Dih}(10)$. Very similar arguments show that $C_{G}(t) \cap C_{G}\left(x_{2}\right)=L_{2} \cong \operatorname{Dih}(30)$, and therefore $X_{5 B}$ breaks into two $C_{G}(t)$-orbits of sizes $2^{7} \cdot 3^{4} \cdot 5 \cdot 7 \cdot 11$ and $2^{7} \cdot 3^{3} \cdot 5 \cdot 7 \cdot 11$.

Since we have now dealt with all the non-empty subsets $X_{C}$, the proof of Theorem 2.2 is complete.

\subsection{Calculations in $E_{6}(2)$}

We now look further at the calculation in $E_{6}(2)$, mentioned in Section 1. Repeating the calculation for the given $t$ and $s 100$ times, in each case using 10,000 random elements, gave the following five outcomes with the following frequencies.

\begin{tabular}{llllll}
\hline$|\langle S\rangle|$ obtained & $2^{12} \cdot 3 \cdot 7$ & $12^{2} \cdot 3$ & $2^{11}$ & $2^{10} \cdot 3$ & $2^{10}$ \\
\hline Number of times & 69 & 20 & 2 & 4 & 5 \\
\hline
\end{tabular}

On the other hand, using 12,000 random elements from $E_{6}(2)$ in each of 100 experiments always gave $|\langle S\rangle|=2^{12} \cdot 3 \cdot 7$.

\subsection{Calculations in $E_{8}(2)$}

We again go to [24] for our copy of $G=E_{8}(2)$, using the 2-generator version there. So $G=\langle a, b\rangle$ with $a$ and $b$ being $248 \times 248$ matrices over GF(2). Let $V$ 
denote the 248-dimensional GF(2) $G$-module. The order of $a$ is 30 and the order of $b$ is 10 . We shall employ the naming system in [1] (which follows ATLAS conventions) for the conjugacy classes of $G$. So $a^{15} \in 2 D$ and $b^{5} \in 2 B$ (because $\operatorname{dim} C_{V}\left(a^{15}\right)=128$ and $\left.\operatorname{dim} C_{V}\left(b^{5}\right)=156\right)$. In each of the following cases, we did 10 experiments each using 40,000 random elements from $E_{8}(2)$.

(i) $t=a^{15}$ and $s=t^{g}$, where $g=b^{3} a b^{-1} a b a^{11}$. Then $t s$ has order 17 with $\operatorname{dim} C_{V}(t s)=32$, so, using [1], $t s \in 17 A B$ with $C_{G}(t s) \cong 17 \times \Omega_{8}^{-}(2)$.

\begin{tabular}{lllllllllll}
\hline$|S|$ & 8 & 5 & 7 & 7 & 4 & 6 & 7 & 3 & 9 & 10 \\
\hline$|\langle S\rangle|$ & $2^{8} \cdot 5$ & $2^{6} \cdot 3$ & $2^{9} \cdot 3$ & $2^{9} \cdot 3$ & $2^{6} \cdot 3$ & $2^{8} \cdot 3$ & $2^{9} \cdot 3$ & $2^{4} \cdot 3$ & $2^{8} \cdot 3$ & $2^{9} \cdot 3$ \\
\hline
\end{tabular}

(ii) $t=b^{5}$ and $s=t^{g}$, where $g=a b^{3} a^{7} b^{4} a^{19}$. This time $t s$ has order 8 .

\begin{tabular}{lllllllllll}
\hline$|S|$ & 78 & 78 & 79 & 68 & 92 & 53 & 75 & 63 & 74 & 91 \\
\hline$|\langle S\rangle|$ & $2^{18}$ & $2^{18}$ & $2^{21}$ & $2^{23}$ & $2^{30}$ & $2^{22}$ & $2^{23}$ & $2^{21}$ & $2^{19}$ & $2^{26}$ \\
\hline
\end{tabular}

In case we are getting too rosy a picture, we have the following.

(iii) $t=b^{5}$ and $s=t^{g}, g=a b^{3} a^{7}$. Here $t s$ has order $5, \operatorname{dim} C_{V}(t s)=68$. So $t s \in 5 A$ with $C_{G}(t s) \cong 5 \times \Omega_{12}^{-}(2)$. Each of the 10 experiments yielded no elements of $S$ at all. Moreover, note that, by Lemma 2.1, $C_{G}(t) \cap C_{G}(s)$ must be non-trivial.

\subsection{Centralizers in a $670 \times 670$ matrix group}

Using the GF(2) representation of $O_{10}^{+}(2)$ of degree 670 given in [24], we may regard $G=O_{10}^{+}$(2) as a subgroup of $\mathrm{GL}_{670}$ (2). In the notation of [24], $G=\langle a, b\rangle$, where $a$ has order 2 and $b$ has order 20 . Set $H=\left\langle a, a^{b}, a^{\left(b a b^{3}\right)}\right\rangle$. We have that $H \cong L_{3}(2)$, and we seek to calculate $C_{G}(H)$. First employing the procedure in Section 1 , we calculate $C_{G}\left(\left\langle a, a^{b}\right\rangle\right)$ (which has order $\left.2^{7} \cdot 3^{3} \cdot 5 \cdot 7\right)$. Then using random elements selected from $C_{G}\left(\left\langle a, a^{b}\right\rangle\right)$, we repeat the procedure to calculate elements in $C_{C_{G}\left(\left\langle a, a^{b}\right\rangle\right)}\left(a^{\left(b a b^{3}\right)}\right)$ (and note that $a^{\left(b a b^{3}\right)} \notin C_{G}\left(\left\langle a, a^{b}\right\rangle\right)$ ), thus delivering a subgroup of $C_{G}(H)$ of order $2^{2} \cdot 3^{2}$. There is of course the perennial problem of knowing whether we have obtained all of $C_{G}(H)$ - though there are instances where just having some elements of $C_{G}(H)$ may be helpful. As it happens, $\left|C_{G}(H)\right|=2^{2} \cdot 3^{2}$ (which may be checked by working in the 496 degree permutation representation for $O_{10}^{+}(2)$ available in [24]). Finally, we remark that the standard MAGMA functions could not calculate $C_{G}(H)$. 


\section{Bibliography}

[1] A. Aubad, J. Ballantyne, A. McGaw, P. Neuhaus, J. Phillips, P. Rowley and D. Ward, The Semisimple Elements of $E_{8}(2)$, MIMS EPrint (2016), http://eprints . maths.manchester.ac.uk/id/eprint/2457.

[2] J. Ballantyne, C. Bates and P. Rowley, The maximal subgroups of $E_{7}(2), L M S J$. Comput. Math. 18 (2015), no. 1, 323-371.

[3] C. Bates, D. Bundy, S. Hart and P. Rowley, Commuting involution graphs for sporadic simple groups, J. Algebra 316 (2007), no. 2, 849-868.

[4] C. Bates and P. Rowley, Involutions in Conway's largest simple group, LMS J. Comput. Math. 7 (2004), 337-351.

[5] C. Bates, P. Rowley and P. Taylor, Involutions in the automorphism groups of small sporadic simple groups, Algebra 2015 (2015), Article ID 587629.

[6] H. Bäärnhielm, D. Holt, C. R. Leedham-Green and E. A. O’Brien, A practical model for computation with matrix groups, J. Symbolic Comput. 68 (2015), no. 1, 27-60.

[7] J. N. Bray, An improved method for generating the centralizer of an involution, Arch. Math. (Basel) 74 (2000), no. 4, 241-245.

[8] J. N. Bray and H. Bäärnhielm, A new method for recognising Suzuki groups, J. Algebra 493 (2018), 483-499.

[9] J. J. Cannon and C. Playoust, An Introduction to Algebraic Programming with Magma (Draft), Springer, Berlin, 1997.

[10] J. H. Conway, R. T. Curtis, S. P. Norton, R. A. Parker and R. A. Wilson, Atlas of Finite Groups, Oxford University, Eynsham, 1985.

[11] H. Dietrich, C. R. Leedham-Green and E. A. O'Brien, Effective black-box constructive recognition of classical groups, J. Algebra 421 (2015), 460-492.

[12] J. D. Dixon, C. E. Praeger and A. Seress, Strong involutions in finite special linear groups of odd characteristic, J. Algebra 498 (2018), 413-447.

[13] A. Farooq, S. Norton and R. A. Wilson, A presentation of the monster and a set of matrices which satisfy it, J. Algebra 379 (2013), 432-440.

[14] W. M. Kantor and K. Magaard, Black box exceptional groups of Lie type II, J. Algebra 421 (2015), 524-540.

[15] R. Lyons, Evidence for a new finite simple group, J. Algebra 20 (1972), 540-569.

[16] P. McMullen and E. Schulte, Abstract Regular Polytopes, Encyclopedia Math. Appl. 92, Cambridge University, Cambridge, 2002.

[17] W. Meyer, W. Neutsch and R. Parker, The minimal 5-representation of Lyons' sporadic group, Math. Ann. 272 (1985), no. 1, 29-39. 
[18] S. Norton, The construction of $J_{4}$, in: Proceedings of the Santa Cruz Group Theory Conference, Proc. Sympos. Pure Math. 37, American Mathematical Society, Providence (1980), 271-277.

[19] S. P. Norton and R. A. Wilson, A correction to the 41-structure of the Monster, a construction of a new maximal subgroup $\mathrm{L}_{2}(41)$ and a new Moonshine phenomenon, J. Lond. Math. Soc. (2) 87 (2013), no. 3, 943-962.

[20] C. Parker and R. Wilson, Recognising simplicity of black-box groups by constructing involutions and their centralisers, J. Algebra 324 (2010), no. 5, 885-915.

[21] P. Rowley and P. Taylor, Involutions in Janko's simple group $J_{4}, L M S$ J. Comput. Math. 14 (2011), 238-253.

[22] C. C. Sims, The existence and uniqueness of Lyons' group, in: Finite groups '72, North-Holland Mathematics Stud, 7, North Holland, New York (1973), 138-141.

[23] P. Taylor, Computational investigations into finite groups, $\mathrm{PhD}$ Thesis, University of Manchester, 2011.

[24] R. Wilson, P. Walsh, J. Tripp, I. Suleiman, R. Parker, S. Norton, S. Nickerson, S. Linton, J. John and R. Abbot, Atlas of finite group representations. Version 3, http://brauer.maths.qmul.ac.uk/Atlas/v3/.

[25] R. A. Wilson, Classification of subgroups isomorphic to $\mathrm{PSL}_{2}$ (27) in the Monster, LMS J. Comput. Math. 17 (2014), no. 1, 33-46.

[26] R. A. Wilson, Every $\mathrm{PSL}_{2}(13)$ in the Monster contains 13 A-elements, LMS J. Comput. Math. 18 (2015), no. 1, 667-674.

Received March 22, 2019.

\section{Author information}

Veronica Kelsey, Department of Mathematics, University of St Andrews, St Andrews, Great Britain.

E-mail: veronicakelsey@live.com

Peter Rowley, Department of Mathematics, University of Manchester, Oxford Road, Manchester, United Kingdom.

E-mail: peter .j.rowley@manchester .ac.uk 